\title{
Poisson Distribution with Discrete Parameter
}

\author{
$1^{\text {st }}$ Dina Fitria \\ Mathematics Department \\ Universitas Negeri Padang \\ Padang, Indonesia \\ dinafitria@fmipa.unp.unp.ac.id
}

\author{
$2^{\text {nd }}$ Nonong Amalita \\ Mathematics Department \\ Universitas Negeri Padang \\ Padang, Indonesia \\ nongmat@fmipa.unp.ac.id
}

\author{
$3^{\text {rd }}$ Syafriandi \\ Mathematics Department \\ Universitas Negeri Padang \\ Padang, Indonesia \\ syafriandi_mat@fmipa.unp.ac.id
}

\begin{abstract}
Construction of distribution is an interesting work for statistician and mathematician. The most powerful distribution for discrete case is Poisson with a specified parameter, usually a constant. In this case, we study the Poisson distribution for which its parameter is also a discrete random variable. We found that the Poisson distribution with discrete parameter has different characteristic, e.g. distribution function, mean, variance and others statistics. Its distribution function different with the other known distribution and show complex form.
\end{abstract}

\section{Keywords-Discrete, Poisson, Random Variable}

\section{INTRODUCTION}

An event is Poisson random variable $(X)$ with a constant parameter $\lambda$, tells the number of outcomes in an interval or event in term of $t$ with probability function

$$
p(x, \lambda t)=\frac{e^{-\Delta t}(\lambda t)^{x}}{x !}, x=0,1,2, \ldots
$$

with $E(X)=\operatorname{Var}(X)=\lambda t$. Poisson distribution is limiting distribution of binomial when its parameter $\lambda$ tends to small and $n$ is too large.

When we randomize the parameter $\lambda$ by some probability on $(0, \infty)$, we find the new distribution function Mixed Poisson on the set of nonnegative integers $\mathbb{N}$. It distribution function defined by [1]

$$
F()=\int_{0} F(\cdot \mid \lambda) d G(\lambda)
$$

where $G(\lambda)$ is distribution function of parameter $\lambda, \lambda \in \Theta$.

In term of probability function, if $X \mid \Lambda \sim \operatorname{Poi}(A)$ and $A \sim g(\lambda)$, the unconditional probability function $X \sim \operatorname{MPoi}(\lambda)$ for which

$f(x)=\int_{0} f(x \mid \lambda) g_{\lambda}(\lambda) d \lambda$

The probability function of $g($.$) is referred as mixing density$ if $g($.$) is continuous [2] or finite mixture if g($.$) is discrete$ [3]. Generally, all distribution function of Poisson random variable for which $\lambda$ is also random variable called Mixed Poisson distribution.

There are some research about Mixed Poisson processes. Most of the research used Gamma distribution as mixing distribution of Poisson.[1], [4], [5]. Actually, it gives the fact that unconditional distribution of Poisson if its mixing distribution $\Gamma$ distribution, is negative binomial. It accommodate the weak side of Poisson with random parameter, having over-dispersion properties. In other research, [6] shows the other mixing distribution such as Invers Gaussian, log normal. Most of mixing distribution used are continuous random variables. [7] used some discrete mixture like zero modified distribution. In this research, we study how if the mixing distribution is discrete random variables. They are Binomial, Poisson and Geometric distribution.

\section{MIXED POISSON}

In the history of mixed Poisson, it started by the work of Lundberg on 1940 in his thesis with title on random processes and their application to sickness and accident statistics. He introduced the term compound Poisson as mixed Poisson we know nowadays. In 1964 he introduced compound Poisson which are different to the previous one. After that, the word mixed Poisson is used as a process of Poisson with a random variable parameter. In other reference, mixed Poisson is also known as weighted Poisson [4].

Define $f(x \mid \lambda) \wedge g(\lambda)$ as mixed distribution with mixing distribution $g()$ And define $f(x \mid \lambda), g(\lambda \mid \mu), h(\mu)$ on the same structure. We find that mixed distribution are [1]:

\section{Associative}

$[f(x \mid \lambda) \wedge g(\lambda \mid \mu)] \wedge h(\mu) \quad$ is $\quad$ equivalent $\quad$ to
$f(x \mid \lambda) \wedge[g(\lambda \mid \mu) \wedge h(\mu)]$

2. Expected value

$$
E[h(X)]=\int_{0} E_{x \mid \lambda}[h(X)] g(\lambda) d \lambda
$$

3. Mean

$$
E[X]=E\left[E_{x] a}(X)\right\rfloor
$$

4. Variance

$$
\operatorname{Var}[X]=\operatorname{Var}\left[E_{x \mid \lambda}(X)\right]+E\left[\operatorname{Var}_{x \mid \lambda}(X)\right]
$$

5. Distribution of product of two random variables being the same as the mixing distribution

And mixed Poisson properties are associative and overdispersion. Actually let $X \mid A \sim P o i(A)$ and $A$ is a mixing distribution with specified parameter, called $\mu$. So, mean of $X$ will be

$$
E(X)=E\left[E_{x \mid \lambda}(X)\right]=E(\mu)
$$

and variance will be 


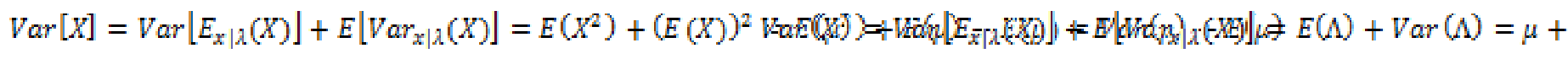

Mixed Poisson has a higher probability to the event $X=0$ and has a longer tail than simple Poisson distribution.

We can construct mixed Poisson distribution by direct calculation, recursive formula, Laplace transformation technique or using a special function.

\section{POISSON DISTRIBUTION WITH DISCRETE PARAMETER}

We use binomial, Poisson and geometric distribution as mixing distribution of Poisson. Binomial distribution separate the events into two parts, "yes" and "no", or "right" and "wrong", and so on.

Random variable $X$ is Binomial distributed with parameter "success" $p$ and "fails" $1-p$, if it probability function

$f(x)=\left(\begin{array}{l}n \\ p\end{array}\right) p^{x}(1-p)^{n-x}$ for $x=0,1_{x \ldots}, n$ and $f(x)=0$ elsewhere. When we assume that $X \mid \Lambda \sim P o i(\Lambda)$ and $A \sim B$ in $(p)$ so we have

$$
\begin{gathered}
f(x)=\sum_{\lambda=0}^{\infty} f(x \mid \lambda) g(\lambda)=\sum_{\lambda=0}^{\infty} \frac{e^{-\lambda} \lambda^{x}}{x !}\left(\begin{array}{l}
x \\
\lambda
\end{array}\right) p^{\lambda}(1-p)^{x-\lambda}=\sum_{\lambda=0}^{\infty} \\
=\frac{n !}{x !} \sum_{\lambda=0}^{\infty} \frac{e^{-\lambda} \lambda^{x}}{(n-\lambda) ! \lambda !} p^{\lambda}(1-p)^{n-\lambda}
\end{gathered}
$$

Statistical properties of mixed Poisson-binomial

a. Mean

$$
E(X)=\int_{0} E_{x \mid \lambda}(X) g(\lambda) d \lambda=E(A)=n p
$$

Because $A \sim \operatorname{Bin}(n, p)$ we have $E(A)=n p$

b. Variance

$$
\operatorname{Var}(X)=\operatorname{Var}\left[E_{x \mid \lambda}(X)\right]+E\left[\operatorname{Var}_{x \mid \lambda}(X)\right]=E(A)+\operatorname{Var}(\lambda)=n p(2-p)
$$

Based on variance of $\operatorname{Binomial}(n, p)$ which is $n p(1-p)$. Based on mean and variance we also have that mixed Poisson-binomial is over dispersion, for which variance having bigger value than mean.

How if the parameter of Poisson in also Poisson distributed. $\quad X \mid \Lambda \sim P o i(\Lambda)$ and $\Lambda \sim P o i(\mu)$. So unconditional distribution function of $X$ will be

$$
f(x)=\sum_{\lambda=0}^{\infty} f(x \mid \lambda) g(\lambda)=\sum_{\lambda=0}^{m} \frac{e^{-\lambda} \lambda^{x}}{x !} \times \frac{e^{-\mu_{\mu} \mu^{\lambda}}}{\lambda !}=\sum_{\lambda=0}^{m} \frac{e^{-(\lambda+\mu)} \lambda\left(x^{4} \mu^{x}\right.}{x ! \lambda !}
$$

Mixed Poisson-Poisson has mean as follows

$$
E(X)=E(\Lambda)
$$

Known that mean of random variables $X \sim P o i(\lambda)$ is $E(X)=\lambda=\operatorname{Var}(X)$

So, mixed Poisson-Poisson having mean

$$
E(X)=\int_{\Lambda} E_{x \mid \lambda}(X) g(\lambda) d \lambda=E(\Lambda)=\mu
$$

And variance
The authors are grateful to the Universitas Negeri Padang reviewer's constructive for funding the research.

It's clear explain that $E(X)>\operatorname{Var}(X)$.

In other cases, for $X \sim G e o(p)$ with distribution function $f(x)=p(1-p)^{x-1}$. If $X \mid A \sim P o i(A)$ and $A \sim G e o(p)$ so distribution function of mixed Poisson-Geometric can be ormulate by

$$
f(x)=\sum_{\lambda=0}^{\infty} f(x \mid \lambda) g(\lambda)=\sum_{\lambda=0}^{\infty} \frac{e^{-\lambda} \lambda^{x}}{x !} p(1-p)^{\lambda-1}
$$

A geometric distribution having mean $\frac{1}{p}$ and variance $\frac{1-p}{p^{2}}$.

Mixed Poisson-Geometric will be overdispersion by mean

$E(x)=\frac{1}{p}$

And variance

$\operatorname{Var}(X)=E(X)+\operatorname{Var}(A)=\frac{1}{p}+\frac{1-p}{p^{2}}=\frac{1}{p^{2}}$

Bigger the lparameter of $A$ less the mean and variance of it daPoisson with discrete mixture can accommodate events are counted as zero. We also conclude that Poisson distribution with discrete parameter or known as discrete mixed Poisson having the properties which are different with traditional Poisson. It depends on its mixture distributions. the future research we can study the properties of mixture of mixed discrete and continuous distribution. Is it possible to accommodate the underestimate case or not.

\section{ACKNOWLEDGMENT}

[1] D. Karlis and E. Xekalaki, "Mixed Poisson Distributions," Int. Stat. Rev., vol. 73, no. 1, pp. 35-58, 2005.

[2] R. J. Sarguta, "On The Construction of Mixed Poisson Distributions," no. July, 2012

[3] U. . M. Titterington, D.M., A.F.M Smith, "Statistical Analysis of Finite Mixture Distributions." Wiley Series on Probability and Mathematical Statistics, 1985

J. Grandell, Mixed Poisson Processes, Monongraph. SpringerScience+Business Media, B.V, 1997.

D. Fitria, "Banyak Klaim Asuransi : Model dan Sifat-sifat Statistik," Master Thesis, 2013.

J.-F. W. Denuit, Michel, Xavier Marechal, Sandra Pitrebois, Actuarial Modelling of Claim Counts. John Wiley\&Sons, Ltd, 2007.

N. L. Johnson, A. W. Kemp, and S. Kotz, Univariate Discrete Distributions, vol. 17, no. 2. 2005. 